\title{
Cylindrical flowing-junction cell for the investigation of fluctuations and pattern-formation in miscible fluids
}

Cite as: Rev. Sci. Instrum. 90, 085109 (2019); doi: 10.1063/1.5112778

Submitted: 3 June 2019 - Accepted: 5 August 2019 •

Published Online: 23 August 2019

\author{
Fabrizio Croccolo, , a) (D) Doriano Brogioli, ${ }^{2}$ (D) and Alberto Vailati ${ }^{3}$

\begin{abstract}
AFFILIATIONS
${ }^{1}$ Laboratoire des Fluides Complexes et leurs Réservoirs (LFCR), IPRA, UMR5150 E2S-Univ Pau \& Pays Adour, CNRS, TOTAL, 64600 Anglet, France

${ }^{2}$ Universität Bremen, Energiespeicher-und Energiewandlersysteme, Bibliothekstraße 1, 28359 Bremen, Germany

${ }^{3}$ Dipartimento di Fisica Aldo Pontremoli, Università degli Studi di Milano, I-20133, Milano, Italy
\end{abstract}

a) Electronic mail: fabrizio.croccolo@univ-pau.fr

\begin{abstract}
We describe a flowing-junction cell with cylindrical symmetry suitable to investigate fluctuations and pattern formation at the diffusing interface between two miscible phases of a liquid mixture. The continuous outflow of the remixed fluid through a thin slit located at the midheight of the sample allows the preparation of an initially sharp interface. The system can be used in both gravity-stable and unstable conditions. In the stable case, the denser liquid is on the bottom of the cell and mass diffusion is the only active process for remixing the two liquids. Once the flow is stopped, one can investigate nonequilibrium fluctuations during free-diffusion in a binary mixture or double diffusive instabilities in multicomponent mixtures. Two horizontal transparent windows allow vertical mapping of the fluid flow by using shadowgraphy. In the unstable condition, with the denser fluid on top, stopping the radial flow at the interface gives rise to a RayleighTaylor instability, which drives the denser liquid toward the bottom of the cell. The fact that the cell can maintain the system in the unstable condition shows that it is suitable to perform experiments under microgravity conditions. With respect to other free-diffusion cells, the proposed configuration has the advantage that the interface is extremely stable and flat, and that the experiments can be repeated by just flowing the cell with fresh liquids.
\end{abstract}

Published under license by AIP Publishing. https://doi.org/10.1063/1.5112778

\section{INTRODUCTION}

The behavior of complex fluids is commonly studied by means of noninvasive optical techniques, which allow one to retrieve the variations of the refractive index of the fluid, and in turn, the variations of the density of isothermal fluids. An important system is represented by a nonequilibrium fluid stratified by the presence of a macroscopic gradient, such as a composition gradient. Several experimental setups have been devised to observe the fluid sample from one side, in a direction perpendicular to the gradient, in order to retrieve the evolution of the vertical profile of the sample. This is done by using optical methods to investigate the temporal evolution of the concentration profile of a binary mixture of two miscible fluids as a function of the vertical coordinate and time in order to measure the mass diffusion coefficient by means of different techniques, like interferometry, ${ }^{1,2}$ beam deflection, ${ }^{3,4}$ electronic speckle pattern interferometry, ${ }^{5,6}$ and optical digital interferometry. ${ }^{7}$ Hele-Shaw cells with lateral optical access in combination with optical methods are used to investigate diffusive instabilities in unstable fluid layers ${ }^{8-12}$ and chemical instabilities in reacting systems. ${ }^{13-15}$ Instabilities have also been observed by using Gouy interferometry. ${ }^{16-19}$

A different approach is that of observing the fluid sample vertically, in the direction parallel to the gradient; ${ }^{20}$ in this case, one is no more sensitive to the vertical profiles but can investigate the $2 \mathrm{D}$-spatial variations of the fluid thermodynamic variables in the horizontal plane. This is the approach frequently adopted for the investigation of pattern formation in convecting fluids under 
the action of a temperature gradient ${ }^{21}$ and for the investigation of nonequilibrium fluctuations determined by the presence of a macroscopic concentration gradient under isothermal conditions. ${ }^{22-27}$

In all the experimental approaches mentioned above, the optical apparatus needs to be combined to a device that provides the desired optical access and, at the same time, allows the preparation of the fluid in a well-controlled initial nonequilibrium condition, which typically includes a precise temperature control. In this paper, we will deal with the investigation of isothermal mixtures layered by the presence of a concentration gradient. In particular, we will focus onto the configuration where two miscible phases of a liquid mixture are brought into contact to create an initially sharp interface between them. The preparation of a flat interface between two miscible liquid phases can be achieved by layering them in the presence of the terrestrial gravitational field, with the lighter fluid over the denser one. ${ }^{22,23,26}$ In the case of ternary mixtures, a double-diffusive convective instability can be triggered notwithstanding the presence of an initially stabilizing density profile. Conversely, in the case of binary liquid mixtures, buoyancy keeps the system stable so that, in the absence of thermal gradients, no convective motion occurs and mass diffusion is the only mechanism driving the system toward a totally homogeneous state. In a typical free-diffusion experiment, the sample is prepared in a glass cuvette, which is typically of rectangular shape, and light is shone through the sample from the lateral side. A suitable optical diagnostics allows recording the information about the concentration profile as a function of the height. This kind of configuration allows an accurate measurement of the diffusion coefficient from the time evolution of the macroscopic concentration profile.

The use of the diffusion cell where the concentration gradient is parallel to the direction of observation allows the investigation of a different class of phenomena. In fact, under this configuration, the propagation of light is not affected by the macroscopic concentration gradient and is sensitive only to the perturbations determined by it. As stated above, this approach has been used to investigate nonequilibrium fluctuations generated by a concentration gradient. ${ }^{22-27}$ The analysis of the mean squared amplitude of nonequilibrium fluctuations and of their dynamics allows one to recover information about the transport processes taking place in the system. ${ }^{28}$

Another experiment that can take advantage of a similar preparation of the sample is the so-called Rayleigh-Taylor Instability (RTI). This kind of instability takes place whenever a denser fluid layer is layered over a lighter one within the gravitational field. In this case, the buoyancy force induces a large convective motion that brings the denser fluid downward and the lighter one upward resulting in a complete inversion of the sample. In this case, experiments are typically performed in rectangular vessels, mostly $2 \mathrm{D}$ in the so-called Hele-Shaw configuration, ${ }^{11,12}$ with the optical beam passing, again, horizontally through the cell in order to get information about the concentration profile of the investigated fluid.

For all of the abovementioned experiments, the mixture of two miscible fluids is to be prepared in a very difficult-to-achieve initial condition: the two fluids have to be completely separated by a thin and planar interface at some initial zero time. This task is particularly hard to obtain for the RTI experiment, with the denser fluid on top of the lighter one, due to the instability that destabilizes the interface. In a similar way, the preparation of such an initial condition is rather difficult in microgravity conditions or with equal density fluids, due to the lack of the stabilization determined by the buoyancy force, which gives rise to a spontaneous layering of the fluid in the vertical direction. This latter isodensity condition can thus be seen as a transition between the stable case where the density gradient points downward and the unstable case where it points upward. In microgravity, one needs an alternative force to keep the two fluids separated, as is done, e.g., by using surface tension in the case of the cell developed by the group of Kerstin Eckert. ${ }^{8,9}$

Different solutions have been proposed to achieve the preparation of a flat interface between two miscible liquid phases. A first solution is that where the two liquids are kept separated by a thin solid diaphragm, that is pulled away to get the two fluids into contact. ${ }^{29}$ Another solution is the so-called sliding cell where the two halves of the cell can slide one over the other, thus bringing the two fluids into contact. ${ }^{30-33}$ An alternative approach exploits the usage of critical mixtures. ${ }^{24,25}$ If a critical mixture is prepared in the gravitational field at a temperature in which it is phaseseparated, the lighter phase is confined to the top of the vessel by the buoyancy force, while the denser one is forced to the bottom. When the temperature is changed to cross the coexistence curve into a one phase condition, then the mass diffusion process starts. This procedure allows the investigation of nonequilibrium fluctuations, but not RTI and furthermore cannot be used in microgravity conditions.

\section{FLOWING-JUNCTION CELL}

The solution adopted by us to prepare a flat interface between two miscible liquid phases is called Flowing-Junction cell (FJC). Its concept was proposed by Longsworth ${ }^{1}$ in 1950 to investigate diffusion processes in liquids and was further developed by Thomas in the $60 \mathrm{~s} .{ }^{1,2}$ In this device, the two fluids are admitted into the vessel from its opposite sides and flow out from a narrow slit at the central plane of the cell. In this way, while the remixed fluid is removed, the two fluids remain well separated, and the interface between the two is kept sharp. ${ }^{34-41}$

Cells based on the FJC concept have been already used in several diffusion experiments even in microgravity conditions. ${ }^{42,43}$ Former FJCs showed rectangular geometry and were very thin in one of the two horizontal dimensions (quasi-2D configuration). This was done to optimize the removal of the fluid in the mixing region by the larger side of the cell. The interface achievable with a cell with this geometry was of the order of $5 \times 50 \mathrm{~mm}$ and the height of the cell could be varied in the range of some centimeters. This geometry has been proven to be adequate for diffusion coefficient measurements in which the concentration profile was analyzed as a function of the height and time by looking at the cell parallel to the interface. This kind of geometry was utilized more recently in Hele-Shaw-type cells in order to investigate the behavior of fluids undergoing chemical reaction at their interface, also under microgravity conditions. ${ }^{8-12}$ While other methods mentioned above cannot recreate the initial condition iteratively and introduce strong disturbances to the interface between the two fluids, the FJC can be used to easily reset the initial condition in order to iterate the experiment by simply flushing again the fluids and can also work in microgravity or gravitationally unstable conditions. 


\section{A. Cylindrical FJC}

Traditionally, the FJC adopts a lateral view of the sample, perpendicular to the concentration gradient, which allows one to characterize the time evolution of the concentration profile to determine the diffusion coefficient. Conversely, the FJC developed by us adopts a vertical view of the sample in the direction parallel to the concentration gradient. This is the ideal configuration to investigate perturbations of the concentration in the absence of the strong perturbation of the index of refraction determined by the presence of a macroscopic concentration profile. This method provides access to a different group of experiments, with respect to the ones described above, requiring the observation of the variation of the concentration in the direction perpendicular to the concentration gradient. A notable example is the investigation of nonequilibrium fluctuations (NEFs). ${ }^{22,24-28}$ In such cases, the fluid layer is observed by sending a light beam parallel to the density gradient, with a suitable diameter in order to get the desired statistical sample.

From the hydrodynamic point of view, the flow speed needed initially to flush the interface between the two liquid phases must be as homogeneous as possible along the interface; hence, the radial symmetry becomes a natural choice. However, a complete new design is needed with respect to existing schemes because of the difficulty of building a thin slit lying on the interface plane. The FJC presented in this paper exhibits a cylindrical symmetry that enables the radial outflow of the remixed fluid.

The working principle of the cell has been tested first by developing a simple test prototype made of three coaxial Plexiglas cylinders. In Fig. 1, a demonstration test of the cell under operating conditions is shown. In the left picture, the liquids are fluxed and the two incoming liquids with different colors are kept separated despite the unstable condition due to a slight density mismatch and the action of gravity. The red liquid (above) is denser by about $1 \%$ than the green one (below). In the right panel, the image is taken some seconds after stopping the flux. A large convective instability is clearly visible in the cell.
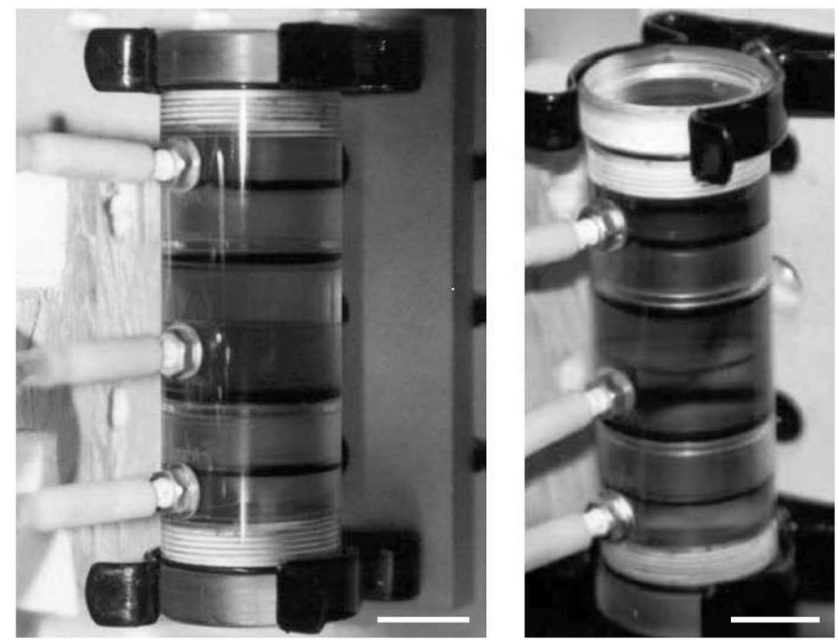

FIG. 1. Images of the FJC cell (left) during flux and (right) some seconds after stopping the flux. The red liquid (top) is slightly denser than the green one (bottom). The white scale-bar corresponds to $15 \mathrm{~mm}$.
In Fig. 2, a visual representation of the flowlines inside the FJC is provided. Obtaining a homogeneous radial flow, free of anisotropies, requires the implementation of several technical details.

The core of the cell is essentially made by two annular metallic flanges (black parts in Fig. 3) with a circular cavity in which a porous ceramic ring is placed (red parts in Fig. 3). The internal faces are separated by a slit as thin as $0.1 \mathrm{~mm}$. This is the narrow slit through which the remixed fluid flows out of the cell. Two annular rings, of the same material, are positioned at the inlet points. The porous ceramic rings have been chosen in order to optimize the radial symmetry of the fluxes. Tests have been extensively performed, e.g., by observing the flux of a fluid slightly warmer than the fluid in the cell entering from one of the cell access, as visible in Fig. 4 (Multimedia view). The top and bottom of the cell are closed by annular plates, which at the same time hold two transparent windows (blue parts in Fig. 3). These windows provide the optical access to the cell. This cell includes also a temperature control made by a water loop (not shown in Fig. 2) coming from a thermostatic bath providing a temperature control within $\pm 0.05 \mathrm{~K}$. The remixing fluids are slowly pushed inside the cell from two slightly pressurized reservoirs, while the flux can be interrupted by three electric-valves (two for inlets and one for outlet). To better explain the working principle of the cell, the fluid path is described (see Fig. 2). Each of the two fluids is pushed from the pressurized reservoir into the cell from top or bottom inlet and fills the volume external to the porous ring. Slowly the fluid permeates the ring and accesses the diffusing chamber starting moving to the central plane of the cell (downward and upward). At this plane, the two fluids come into contact and leave the inner part of the cell through the narrow slit. Eventually, the remixed fluid permeates the exit porous ring and is disposed of via the outgoing tube. In this way, the two liquids remix only at the midplane of the

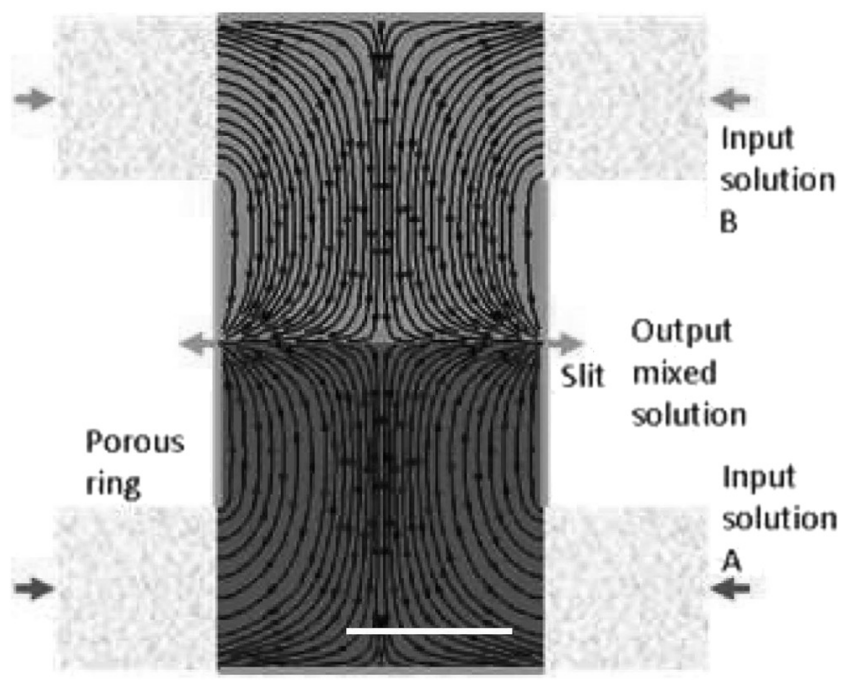

FIG. 2. Scheme of the working principle of a flowing-junction cell. The two fluid phases enter the cell radially through two ceramic rings. The fluids remix at the midheight of the cell. The mixed region is continuously flushed out through a narrow slit until the start of a measurement. The white scale-bar corresponds to $10 \mathrm{~mm}$. 
cell where they meet, but the diffused mixture is rapidly removed through the slit and a flat and tiny interface is maintained midway the cell. When the flow is stopped, this interface can freely evolve and experiments can be performed.

\section{B. Speed distribution}

In order to quantitatively evaluate the efficiency of the removal of the remixed fluid, an experiment was performed to track the fluid speed at the interface plane. A Particle Image Velocimetry (PIV) experiment was performed by using a shadowgraph apparatus and the NE fluctuations as tracker "particles." A sample image of the fluctuations is shown in Fig. 5 (Multimedia view), where one can clearly identify the radial flow of the fluctuations. The experiment was performed by flushing the cell with ethanol $\left(\rho=0.789 \mathrm{~g} / \mathrm{cm}^{3}\right.$ at $\left.20^{\circ} \mathrm{C}\right)$ on the upper side and methanol $\left(\rho=0.792 \mathrm{~g} / \mathrm{cm}^{3}\right.$ at $\left.20^{\circ} \mathrm{C}\right)$ on the bottom side of the cell, thus, in the stable condition, but with two fluids which, at room temperature, are of nearly equal density $\left(\Delta \rho=3 \times 10^{-3} \mathrm{~g} / \mathrm{cm}^{3}\right.$ at $\left.20^{\circ} \mathrm{C}\right)$. A number of 50 images were recorded with a CCD camera and radial cross correlation functions were calculated. The speed of the fluid was obtained for each radial position, by evaluating the maximum in the correlation function

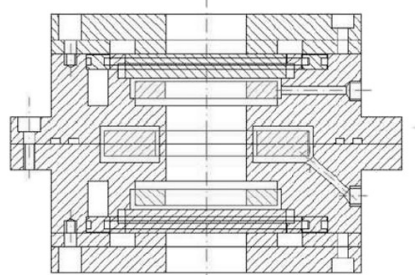

一

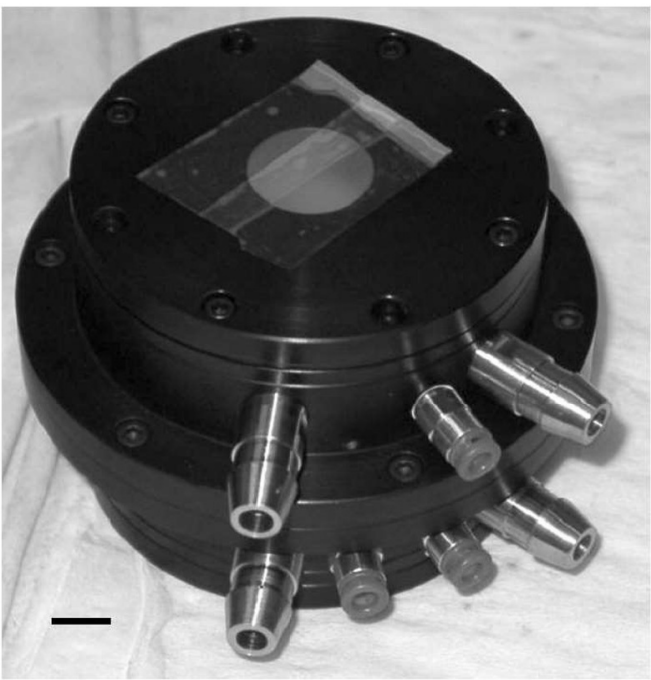

FIG. 3. (Top) Technical drawing and (bottom) picture of the flowing-junction cell The black scale-bar corresponds to $10 \mathrm{~mm}$. thus obtaining the profile of the speed as a function of the distance from the center of the cell (Fig. 6).

Correlation peaks could be obtained in the range $1<r<8 \mathrm{~mm}$; for larger values, the analysis is not feasible because images appear smeared while for lower values, the first maximum in the correlation function overlaps with the natural maximum in zero and it is not possible to distinguish between the two.

For symmetry reasons, the radial speed must vanish for $r \rightarrow 0$. We then approximate the speed as a linear homogeneous relation

$$
u_{r}(r)=a r,
$$

where we assume $a=0.02 / \mathrm{s}$ (the approximation is not shown in the figure). There is a clear discrepancy for small values of $r$ (the experimental data do not point to the origin), which we attribute to difficulties of the measurement.

In order to better justify the choice of the linear relation, we performed a numerical simulation of the system, giving the radial speed
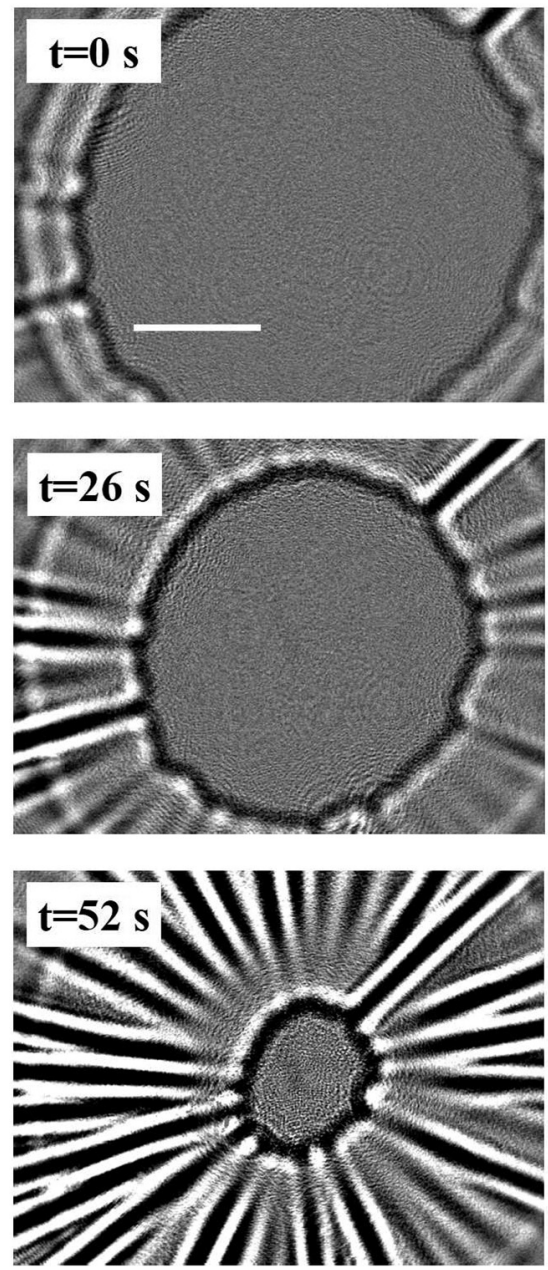

FIG. 4. Images of the filling procedure. A liquid slightly warmer than the one in the cell is slowly injected. The three images are taken at times: (top) $t=0 \mathrm{~s}$, (middle) $t=26 \mathrm{~s}$, and (bottom) $\mathrm{t}=52 \mathrm{~s}$. The white scale-bar corresponds to $5 \mathrm{~mm}$. Multimedia view: https://doi.org/10.1063/1.5112778.1 


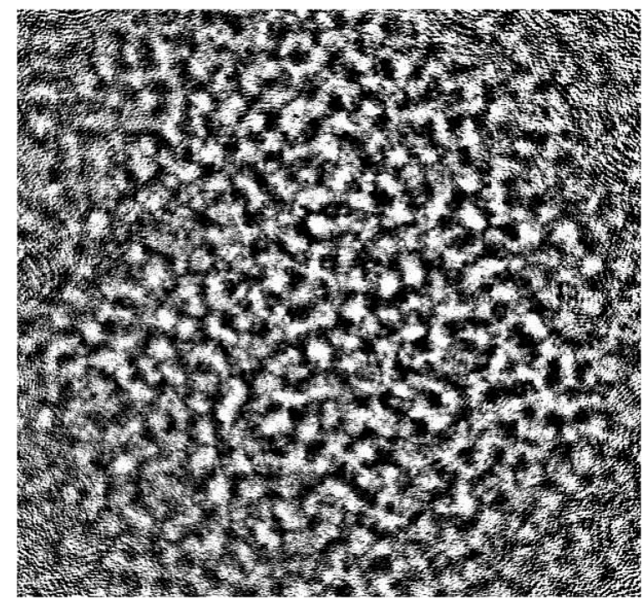

FIG. 5. Shadowgraph image of the nonequilibrium fluctuations in a free-diffusion experiment. Multimedia view: https://doi.org/10.1063/1.5112778.2

$u_{r}$. The model is based on the Navier-Stokes equations, solved by means of a finite element method. The geometry is axially symmetric, with no slip conditions at the walls. The porous inlets are modeled as a fixed constant speed, while the outlet (the slit) is modeled by imposing a pressure jump. The incoming flow is $1.5 \mathrm{~cm}^{3} / \mathrm{min}$.

The resulting $u_{r}$ calculated from the simulation, reported in Fig. 6, solid line, confirms the approximately linear behavior for small $r$, together with a deviation at higher $r$, which is actually observed in the experiment. It is worth noting that the linear dependence of $u_{r}$ on $r$ means that the velocity has a discontinuity of the first derivative at $r=0$, where there is actually a singularity.

Figure 2 shows the flow lines calculated by the simulation. It can be noticed that the flow lines are equally spaced and almost horizontal near the inlets, become approximately axial between the inlets and the outlet slit, and become again horizontal near the plane of the slit.

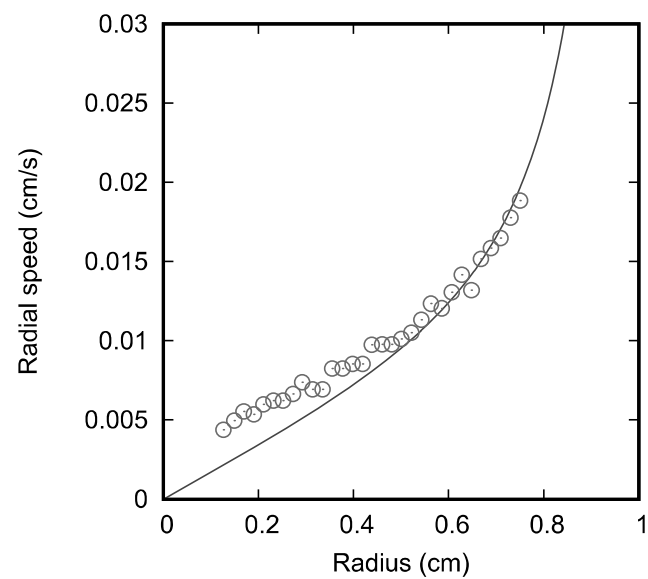

FIG. 6. Graph of the fluid radial speed $u_{r}$ vs radius $r$. The experimental points and the line "model" represent the radial speed on the plane of the slit, measured and calculated, respectively.
Figure 7 shows the speed modulus (norm of the speed) and angle (measured from the plane perpendicular to the axis) calculated by the simulation. From the figure, one can appreciate that the cell is roughly divided into a volume in which the velocity has only axial (vertical) component $u_{z}$ (axial flow volume), and a smaller region close to the interface, midway the cell, where the speed is mostly radial, $u_{r}$ (radial flow disc). The thickness of the radial flow disc reaches its maximum approximately at $r=R / 2$, where the thickness is a few millimeters. It must be noticed that, near the slit, the flow lines are strongly squeezed to enter in the $0.1 \mathrm{~mm}$ space; this explains the strong increase of speed near $r=R$ reported in Fig. 6 .

From the radial speed $u_{r}$, approximated as in Eq. (1), one can derive the transition time required by the liquid to flow from the center of the cell to the exit slit, $t_{t r}$. The radial position of a parcel of liquid, as it moves along the radius, is denoted by $r(t)$. The speed $u_{r}$ at the given $r$ is the time derivative of $r(t)$,

$$
\frac{\mathrm{d}}{\mathrm{d} t} r(t)=u_{r}[r(t)]
$$

By means of Eq. (1),

$$
\frac{\mathrm{d}}{\mathrm{d} t} r(t)=\operatorname{ar}(t)
$$

This equation can be easily integrated,

$$
r(t)=r_{0} e^{a t}
$$

(a) Speed $(\mathrm{mm} / \mathrm{s})$

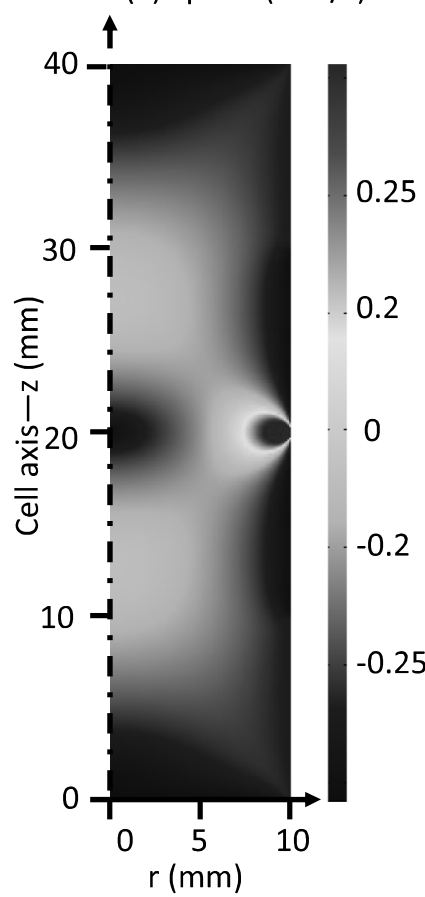

(b) Angle

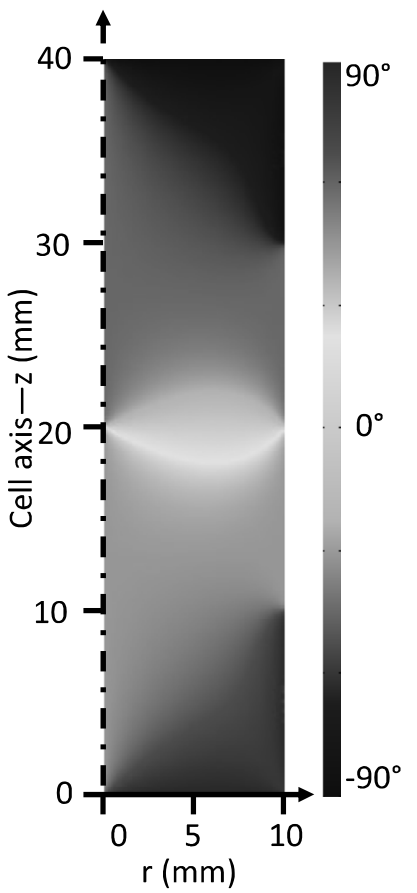

FIG. 7. Graph of the calculated speed. (a) speed modulus (in $\mathrm{mm} / \mathrm{s}$ ), and (b) angle (in degrees from the horizontal plane). 
with arbitrary $r_{0}$. By inverting the relation, we get the time needed to move from a given $r_{0}$ to the exit slit at $r=R$,

$$
t_{t r}=\frac{1}{a} \ln \frac{R}{r_{0}} .
$$

Taking $r_{0}=1 \mathrm{~mm}$, the estimated time is of the order of $100 \mathrm{~s}$. In this time, a diffusion length of $0.3 \mathrm{~mm}$ is reached. This value is much smaller than the thickness of the axial flow volume, of the order of a few millimeters.

\section{Effect of the temperature}

The amplitude of the signal in the case of NE fluctuations is very tiny, in the order of some percent of the average value. Thus, every source of noise should be avoided especially at the interface plane where the concentration gradient is large. In particular, fluctuations in temperature of the order of $50 \mathrm{mK}$ at the interface generate vertical velocity fluctuations and then concentration ones giving rise to disturbances in the order of $2 \%-3 \%$, comparable to the signal of interest. This has been extensively tested and lead us to adopt a temperature control in the form of a circulating water loop from a thermostatic bath with a stability of nearly $\pm 20 \mathrm{mK}$. Also, the two fluid reservoirs were put into the same thermostatic bath and all the inlet tubes were isolated. This allowed an efficient reduction of thermal disturbances while flushing the cell. However, even in this case, thermal oscillations due to the on/off temperature control of the thermostatic bath were visible in the experiment and better results were obtained by stopping the water loop at the same time of the flow of the cell, thus letting the average temperature of the entire system slowly drift toward ambient temperature. However, a more refined temperature control can be envisaged for experiments requiring it; that is, not the case for the example experiments reported here below and is thus beyond the scope of the present work.

\section{RESULTS AND DISCUSSION}

As stated above, the cylindrical FJC allows one to obtain an efficient layering of two miscible or immiscible fluids, either in the stable or unstable condition. More generally, the proposed cell could be a useful tool to study a wide class of mass transport phenomena in isotherm conditions, similar to what have been done for thermodiffusion experiments. ${ }^{28}$ In the following, we present two examples of fluid physics experiments of interest that we have performed taking advantage of the technique. These experiments are, of course, preliminary and systematic studies will be performed in the future. The first experiment is an example of the use of the cylindrical-FJC in the stable case, i.e., layering two miscible fluids with the denser one at the bottom of the cell. This can be quite useful to investigate free diffusion and specifically observe the concentration nonequilibrium fluctuations thus generated during the nonstationary process of diffusion leading to the homogeneous condition when the two fluids are totally remixed. The second experiment is, conversely, an example of the use of our cell in the unstable case so that the denser fluid is now at the top of the cell. We have already tested the efficiency of a prototype in maintaining the unstable condition until the fluid is fluxed as shown in Fig. 1; here, we are interested in the observation of the Rayleigh-Taylor instability by means of the Shadowgraph technique, parallel to the gravity.

\section{A. Nonequilibrium fluctuations in free diffusion}

Mass diffusion takes place in fluid mixtures whenever a concentration gradient is present ${ }^{44}$ giving rise to long-range fluctuations. ${ }^{45,46}$ In the absence of convective motion, diffusion drives the system toward the equilibrium state where the concentration becomes uniform within the sample. ${ }^{47}$ It is commonly believed that diffusion is a homogeneous process at mesoscopic scales. On the contrary, it has been shown that large amplitude fluctuations always take place during the relaxation process. ${ }^{24,27}$ The amplitude of nonequilibrium fluctuations (NEF) is orders of magnitude greater than the thermodynamic equilibrium case. This is due to the coupling of vertical velocity fluctuations with concentration ones given by the macroscopic gradient. Larger fluctuations are greatly enhanced with a $q^{-4}$ dependence ( $q$ being the associated wave vector). This is true only for wave vectors larger than a characteristic $q_{r o}$ depending on fluid properties and the gravity acceleration modulus. For wave vectors smaller than the critical $q_{r o}$, the gravity quenches the fluctuations $s^{24,27}$ and also accelerates them, making fluctuations at $q_{r o}$ being the most persistent in the system. ${ }^{2,28}$ To study the static power spectrum of NEF, the following experimental setup can be used. Here, only a sketch of it is given; more details can be found elsewhere. ${ }^{22,28}$ A Shadowgraph setup is used to record images of the sample and recover the power spectrum by software processing of the images taken at a fixed frame rate. Figure 5 shows a typical image of NEFs once the background (average of 50 images) has been subtracted from the original image and the contrast has been enhanced by a factor of 30 .

In Fig. 8, the NEF power spectrum is shown. The experiment was performed by putting into contact a water/glycerol (at the bottom of the cell) mixture with pure water (at the top of the cell) using the FJC to obtain the initial condition of two separated fluids as described before.

The power spectrum is coherent with the knee shape of NEFs under the influence of gravity. The experiment was performed with a Shadowgraph setup; therefore, for some wave vector range

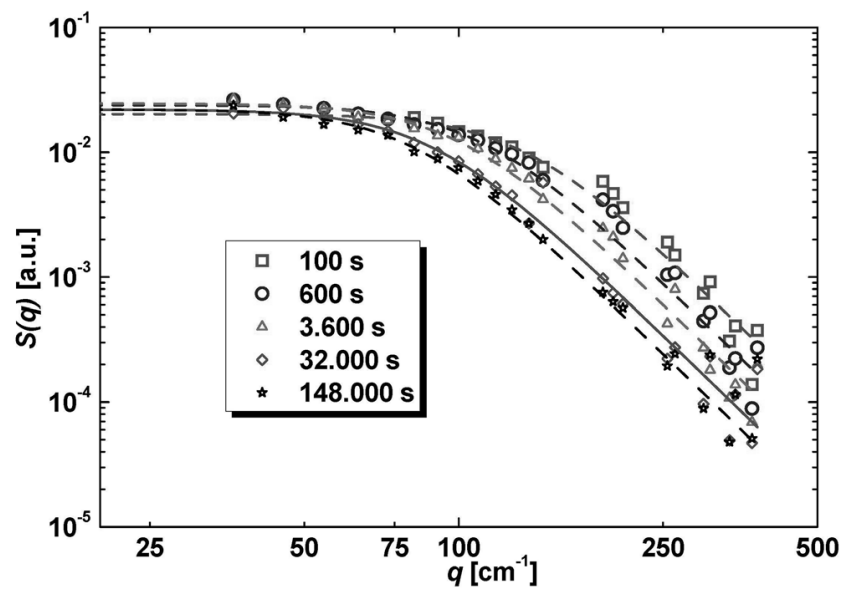

FIG. 8. Time evolution of the static structure factor of nonequilibrium fluctuations for a water/glycerol mixture against pure water. Symbols represent experimental data, while lines are their best fit with the theoretical formula for the spectrum in the presence of gravity. 
data-points are missing due to the poor sensitivity of the technique. The time evolution is as expected and as described in the literature; also, it is possible to observe the $q_{r o}$ decrease as a function of time because of its dependency on the concentration gradient at the interface, which exponentially decreases as diffusion goes by.

\section{B. Rayleigh-Taylor instability}

Rayleigh-Taylor instability (RTI) $)^{11,12,48,49}$ is the collective motion of fluid taking place whenever a high-density fluid is driven by buoyancy force to an underlying layer of a low-density fluid and vice-versa. This instability is of fundamental importance in many applications ranging from fluidized beds, oceans, and atmosphere, to supernovae. ${ }^{50,51}$ While it is typically associated with nonmiscible fluids, it can be observed also in miscible fluids. In the latter case, there is the additional difficulty of preparing the initial condition where the two fluids are layered in the unstable condition while they also remix at the interface.

The FJC design developed by us provides an ideal tool to prepare the mentioned initial condition, thanks to the possibility of layering the two fluids in the unstable condition and the fact that, until the fluid is fluxed within the cell, the remixed fluid at the interface is constantly removed and fresh fluids are put in contact. The result is that a diffusion-free interface is maintained until the flux
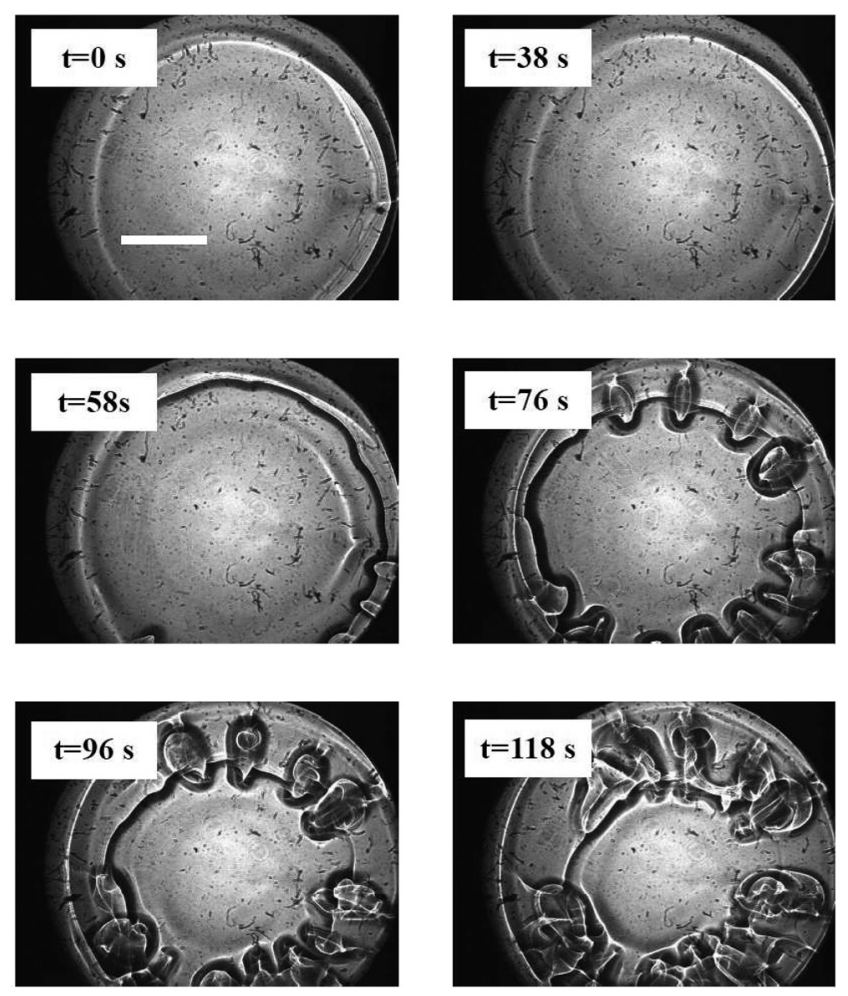

FIG. 9. Observation of Rayleigh Taylor instability in an ethanol-methanol sample. Experimental conditions are: $z=0$, methanol on top, $T=(23 \pm 0.2)^{\circ} \mathrm{C}$. The images are taken at times: $\mathrm{t}=0 \mathrm{~s}, \mathrm{t}=38 \mathrm{~s}, \mathrm{t}=58 \mathrm{~s}, \mathrm{t}=76 \mathrm{~s}, \mathrm{t}=96 \mathrm{~s}$, and $(f) t=118 \mathrm{~s}$. The white scale-bar corresponds to $5 \mathrm{~mm}$. Multimedia view: https://doi.org/10.1063/1.5112778.3 is stopped letting one study the RTI in ideal conditions at the very beginning of the process. This means that one can, in principle, study the pretransitional fluctuations that occur before convection sets in and observe how their amplification due to gravity leads finally to convection. It would be quite interesting to study such fluctuations in the absence of the gravity force, so as to better understand the mechanisms that lead to the development of interfacial fluctuations that trigger RTI, without the masking effect of RTI itself.

In this work, two different experiments are reported where RTI is observed. In Fig. 1, we show a prototype of the cell with transparent walls where the two fluids were water with the addition of dyes and salt. The red liquid contains a small amount of salt so that its density is about $1 \%$ larger than that of the green one. Figure 1(a) is taken while flushing the cell: a stable interface is obtained even if the situation is gravitationally unstable; Fig. 1(b) is taken some seconds after stopping the flux: a large convective cell is apparent in the cell as mark of RTI instability.

A second experiment is performed on a second version of the cell, sharing the same working principle, but made of aluminum and including a temperature control allowing the regulation of the liquid temperature with a precision of $0.1 \mathrm{~K} \mathrm{rms}$ over $1 \mathrm{~h}$. In this case, the two fluids are ethanol and methanol and the experiment is performed at $25^{\circ} \mathrm{C}$ for a density difference of less than $0.5 \%$ and in the unstable condition where methanol is admitted from the top side of the cell. In the sequence of images shown in Fig. 9 (Multimedia view), the flux is stopped some seconds before image (a). The fluid layers quickly remix by convection and the differences of intensity in the images correspond to concentration differences.

\section{CONCLUSIONS}

In this article, a revised version of a flowing-junction cylindrical cell is proposed to study the bulk and fully $3 D$ samples of miscible fluids suitable for performing experiments with a beam of light of a couple of centimeters of diameter passing through the sample vertically, i.e., parallel to the concentration gradient. With this cell, experiments have been performed both in the stable case to study NEF in a free-diffusing sample and in the unstable one to obtain images of the RTI in the vertical direction. We show that this type of cell can be used to prepare thick binary mixture samples with a strong concentration gradient in microgravity conditions.

\section{ACKNOWLEDGMENTS}

We acknowledge the discussion with M. Giglio. F.C. acknowledges financial support from the European Union under the Marie Curie Intra-European Fellowship, Contract No. IEF-251131, the DyNeFI Project, and the French Centre National d'Etudes Spatiales (CNES).

\section{REFERENCES}

${ }^{1}$ L. G. Longsworth, Rev. Sci. Instrum. 21, 524 (1950).

${ }^{2}$ W. J. Thomas and E. McK. Nicholl, Appl. Opt. 4, 823 (1965).

${ }^{3}$ M. Giglio and A. Vendramini, Phys. Rev. Lett. 34, 561 (1975).

${ }^{4}$ T. Triller, H. Bataller, M. M. Bou-Ali, M. Braibanti, F. Croccolo, J. M. Ezquerro, Q. Galand, J. Gavaldá, E. Lapeira, A. Laverón-Simavilla, T. Lyubimova, A. Mialdun, J. M. Ortiz de Zárate, J. Rodríguez, X. Ruiz, I. I. Ryzhkov, V. Shevtsova, S. Van Vaerenbergh, and W. Köhler, Microgravity Sci. Technol. 30, 295 (2018). 